\title{
COMMUNITY-ACQUIRED PNEUMONIA AND SEPSIS CAUSED BY A MULTIRESISTANT STAPHYLOCOCCUS AUREUS STRAIN RESULTING IN A SEVERE AND LONG-LASTING MULTIPLE ORGAN INFLAMMATORY INVOLVEMENT
}

\author{
S. SABBATANI, R. MANFREDI, G. MARINACCI and F. CHIODO \\ Department of Clinical and Experimental Medicine, Division of Infectious Diseases, University of \\ Bologna "Alma Mater Studiorum", S. Orsola Hospital, Bologna, Italy \\ Received March 3, 2006 - Accepted July 14, 2006
}

An exceptional case report of community-acquired, disseminated infection caused by a methicillinresistant Staphylococcus aureus strain, responsible for pneumonia, sepsis, and scattered septic embolism, and accompanied by diffuse polyvisceritis and thrombophlebitis as signs of an overwhelming extensive activation of the immune system, is reported and discussed on the grounds of the most recent evidence in literature. The striking features of a severe, multiresistant $S$. aureus polyvisceral disease associated with multiple immune-mediated focal manifestations are in contrast with the communityacquired origin of infection and the apparent absence of supporting factors for both antimicrobial resistance and the unexpected, exaggerated immune activation occurred in an otherwise healthy man. The extensive immune activation status (as demonstrated by a proportionally maintained absolute lymphocyte cont and a T-cell subset study pointing out an increased quote of CD4+, CD34+, and CD4CD8- cells, compared with a proportional decrease of CD8+ T-lymphocytes) probably had a prominent role in prompting several focal clinical features of our patient, which apparently were not directly related to bacterial invasion, such as the polyvisceritis, the massive pleuric and pericardial effusion, the myocarditis-pericarditis, and the thrombophlebitis occurring in multiple and remote body sites, as compared with to the initial respiratory localization. This case report raises multiple questions regarding the epidemiology, pathogenesis, clinical manifestations, and management of complicated $S$. aureus infection, including the role of novel antimicrobial agents and corticosteroids.

Antibiotic-resistant gram-positive cocci are of increasing concern, especially among hospitalized patients at risk for nosocomial infection (1-3). In particular, both coagulase-positive and coagulasenegative staphylococci represent the largest part of gram-positive cocci affected by rising rates of methicillin resistance, which implies lack of activity of all beta-lactamase derivatives and many other drug classes (1-4). In fact, epidemiological surveys carried out in different settings show a variable but worrying tendency of $S$. aureus isolates to develop methicillin resistance, which may be accompanied by an elevated rate of quinolone resistance and a heterogeneous in vitro activity of glycopeptides (1), and can also lead to an unreliable in vivo effectiveness of these last compounds.

Key words: Staphylococcus aureus, methicillin resistance, pneumonia, sepsis, pleural effusion, myocarditis-pericarditis, hepato-splenic dissemination, multiple thrombophlebitis, immune activation

Mailing address: Prof. Roberto Manfredi,

Infectious Diseases,

University of Bologna,

S. Orsola Hospital,

Via Massarenti 11, I-40138 Bologna, Italy

Tel: +39-051-6363355 Fax: +39-051-343500

e-mail: manfredi@med.unibo.it

117

$1721-727 X(2006)$

Copyright 0 by BIOLIFE, s.a.s.

This publication and/or article is for individual use only and may not be further reproduced without written permission from the copyright holder. Unauthorized reproduction may result in financial and other penalties 
From lower rates $(<2 \%)$ noticed in Scandinavian hospitals to a greater prevalence observed in Mediterranean countries $(>40 \%)$, the phenomenon of methicillin resistance particularly affects bacterial isolates recovered from intensive care, surgery and transplant units where the prevalence may reach over $70-80 \%$ of isolates (3), but may also progressively spread into the community $(2,5)$. While the reenforcement of hospital control measures, rational antibiotic prescribing policies and possibly new antimicrobial and vaccine strategies are searched (6), this phenomenon has an obvious impact on the management of severe infections involving these microrganisms which are related to increased morbidity and mortality rates (6-7) and often need to resort to the last therapeutic resources in this field, represented by the oxazolidinone linezolid $(5,8)$, and the novel streptogramine combination quinupristindalfopristin $(7,9)$. A rare and exemplary case report of community-acquired, severe, disseminated infection caused by a methicillin-resistant $S$. aureus strain and accompanied by diffuse polyvisceritis and thrombophlebitis as signs of a probable extensive immune activation, is reported and discussed on the grounds of the most recent evidence in literature.

A 40-year-old otherwise healthy man, with a mute remote and recent clinical history and not previously treated with antibiotics, was initially hospitalized because of elevated fever, cough, dyspnea, a left pulmonary infiltrate accompanied by a homolateral pleural effusion and a moderate, concurrent pericardial effusion. Despite an initial empiric treatment with ciprofloxacin plus clarithromycin, followed by imipenem, levofloxacin, teicoplanin and fluconazole (pending microbial isolation), the respiratory and general clinical picture rapidly worsened and after three days blood cultures and a broncoscopy with bronchoalveolar lavage allowed the detection of a multiresistant $S$. aureus strain, which consistently showed lack of in vitro susceptibility to methicillin, all beta-lactam derivatives, all macrolides, all fluoroquinolones and all aminoglycosides. A residual antimicrobial activity was retained only by rifampicin $\left(\mathrm{MIC}_{90}\right.$ value $\leq 0.5 \mathrm{mcg} / \mathrm{mL})$, tetracycline $(2 \mathrm{mcg} / \mathrm{mL})$, cotrimoxazole $(\leq 10 \mathrm{mcg} / \mathrm{mL})$, vancomycin $(2 \mathrm{mcg} /$ $\mathrm{mL})$, teicoplanin $(4 \mathrm{mcg} / \mathrm{mL})$, linezolid $(2 \mathrm{mcg} / \mathrm{mL})$, and quinupristin-dalfopristin $(\leq 0.25 \mathrm{mcg} / \mathrm{mL})$. On the basis of the available in vitro sensitivity assays, a combination therapy with full-dose i.v. linezolid plus rifampicin and tetracycline was immediately started but initially it did not contain a further deterioration of the infectious process, which led to an extremely severe and disseminated syndrome involving pulmonary, pleural, miocardial-pericardial and hepato-splenic sites (Figs. 1 and 2), together with a left internal iliac vein and a right popliteal vein thrombophlebitis. Clear signs of overwhelming septic shock (including hyperpyrexia, hypotension, oliguria, toxemia, dyspnea and an acute respiratory distress syndrome) became evident after three days, when intensive care supportive measures were added together with corticosteroid treament. Laboratory examinations showed an increased leukocyte count $(14,760$ cells $/ \mu \mathrm{L}$, with $75 \%$ neutrophils and $20 \%$ lymphocytes), a moderate anemia ( $\mathrm{Hb} 11.8 \mathrm{mmol} / \mathrm{L}$ ), a slight increase of serum transaminase levels (GOT 83 and GPT $57 \mathrm{U} / \mathrm{L}$ ), highly altered serum LDH levels $(2,783 \mathrm{U} / \mathrm{L})$, elevated inflammatory indexes (fibrinogen $2.57 \mathrm{~g} / \mathrm{L}$, ESR 123 first hour, C-reactive protein $21.5 \mathrm{mg} / \mathrm{dL}$ ) and an evident involvement of coagulative parameters (prothrombin time $35 \%$ and D-dimers [fibrin degradation products] $2.74 \mu \mathrm{g} / \mathrm{mL}$ ). After a systematic laboratory study of immune response, a proportionally increased CD3+CD4+ and CD3+CD34+ T-lymphocyte rate was found ( $68 \%$ and $9 \%$ respectively) and a reduced CD3+CD $8+$ percentage was detected $(14 \%)$, as a sign of T-cell immune activation, while CD3 + CD4CD8- cells increased up to a $8 \%$ rate. Finally, no abnormalities were found as to serum complement or immunoglobulin levels, nor to granulocyte chemotaxis and killing properties.

A cardiologic evaluation including ultrasonography diagnosed a myocarditispericarditis, confirmed at a subsequent control performed after one week. A high-resolution computerized tomography (HRCT) of the thorax disclosed multiple, bilateral, scattered parenchimal micronodules (all of them with a diameter below 1 $\mathrm{cm}$ ), suspect of spreading infectious embolism, and confirmed the persistence of an abundant pleuric and pericardic effusion. Functional respiratory tests showed a moderate-severe restrictive ventilation impairment. Swelling and tenderness of the right calf prompted a doppler ultrasonography of the 
lower limbs which disclosed a right popliteal vein thrombophlebitis. A few days later, an abdominalpelvic CT scan showed multiple, disseminated dyshomogeneous hypodense hepatic lesions (predominantly involving II, IV, V, and VII segment) associated with hepatomegaly $(15 \mathrm{~cm}$ maximum diameter) and multiple scattered millimetric spleen infiltrates showing the same characteristic of liver ones, and resembling septic emboli. A frank thrombosis of the left internal iliac vein was concurrently evidenced. A bone marrow biopsy disclosed a hypocellular marrow according to patient's age, with predominant granulocytopoiesis, in the absence of any substitutive or infectious disorder.

After four weeks of treatment with combined linezolid, rifampicin and tetracycline, in the absence of further positive microbiological assays, an empiric ceftazidime plus amikacin therapy was started, but defervescence was achieved and maintained for 8 days only, when fever (up to $40^{\circ} \mathrm{C}$ ) re-appeared. A novel set of cultures did not produce any positive result, so that a predominant dysreactive syndrome was suspected, and high-dose corticosteroid therapy (methylprednisolone $80 \mathrm{mg} /$ day) started together with an antimicrobial association containing i.v.

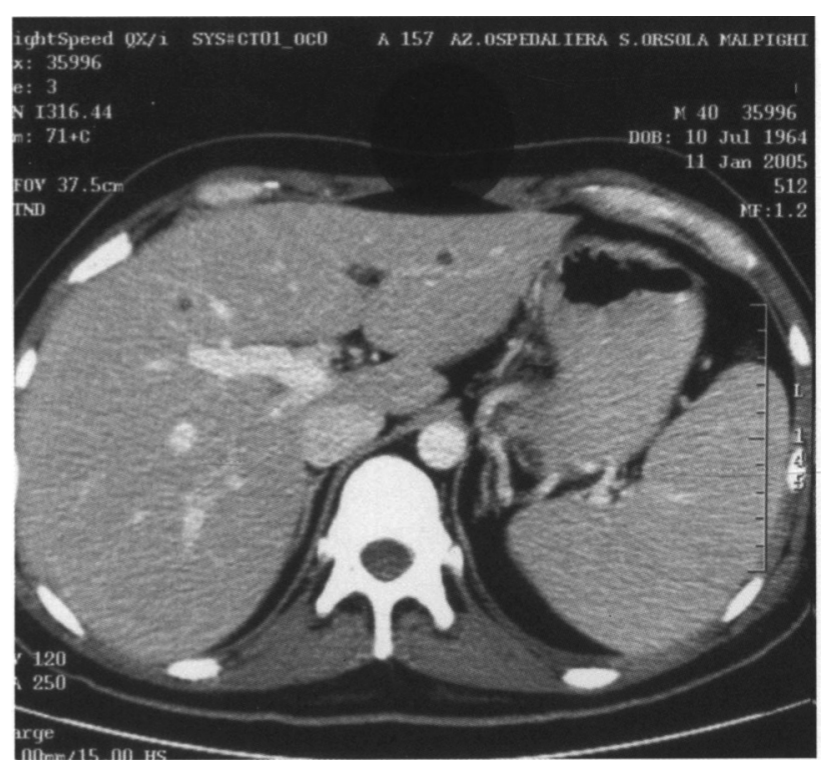

Fig. 1. Multiple septic embolism of both liver and spleen, as evidenced by an abdominal contrast-enhanced $C T$ scan. linezolid, metronidazole, and gentamycin, and anticoagulative and heparin administration for the management of peripheral thrombotic lesions. After ten consecutive days of defervescence, i.v. ticarcillin-clavulanate replaced the prior triple antibiotic regimen, while a slowly progressive decrease of steroid dosage was carried out, during 15 consecutive days. In the meantime, a sonographic doppler study of limbs, repeated three weeks after the first one, showed a significant reduction of thrombophlebitic lesions at both iliac and popliteal sites and an abdominal ultrasonography showed a persistent hepatosplenomegaly, so that a liver biopsy was performed, but histopathologic studies detected a macrovesicular hepatic steatosis, in absence of inflammation or granulomatous lesions. A total-body scintigraphic study with ${ }^{67}$ Ga carried out two months after admission detected residual concentrations of radionuclide at upper pulmonary lobes, and a repeated HRCT scan of the thorax demonstrated a significant improvement of the radiological picture, with disappearance of pleural and pericardial effusion and minimal remaining micronodular parenchimal lesions.

At hospital discharge, after nearly three consecutive months of hospitalization, our patient was afebrile and recovered significantly. A control doppler vein sonography and a cardiac ultrasonography performed two weeks later, showed a complete resolution of thrombophlebitis and myocarditis-pericarditis, respectively. Anticoagulative therapy was interrupted after the doppler vein sonography. An abdominal and pelvic CT scan demonstrated the disappearance of the multiple hepato-splenic parenchimal lesion and the resolution of the iliac vein thrombosis. No other abnormalities occurred during the subsequent careful clinical and laboratory follow-up which continued at monthly intervals for four further months.

\section{DISCUSSION}

From a pathogenetic point of view, it remains widely debated whether or not methicillin resistance confers a greater virulence to staphylococci (regardless of their reduced antimicrobial susceptibility). A recent, extensive cohort study of 815 patients with hospital $S$. aureus infection and bacteremia disclosed 


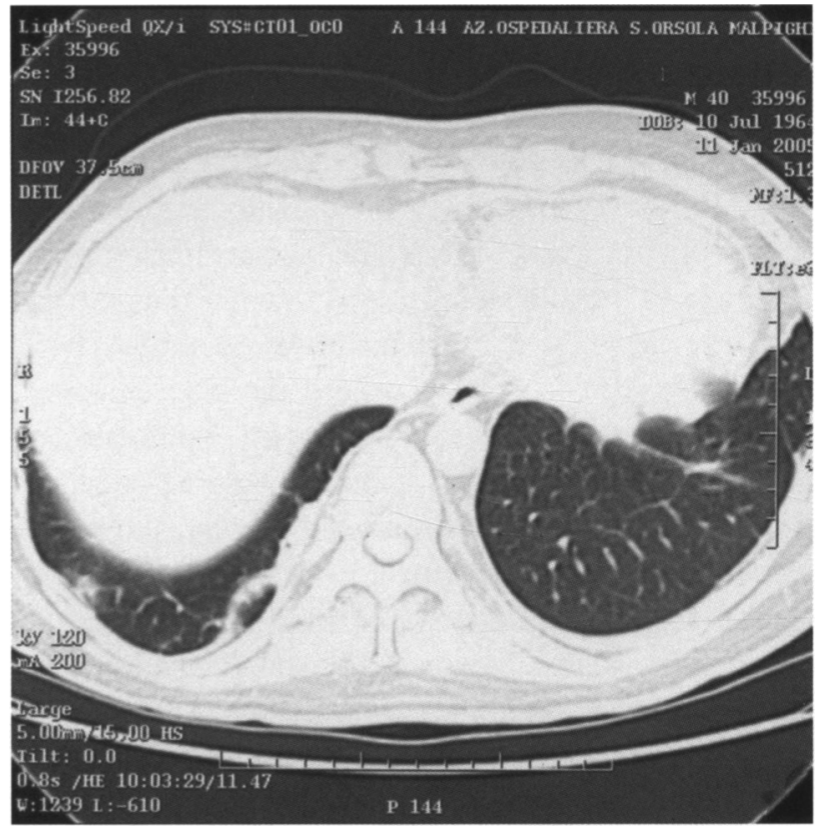

Fig. 2. Extensive pleural effusion accompanying the worsening of pleuro-pulmonary disease, as visible at a contrast-enhanced computerized tomography (CT) scan of the abdomen.

a remarkable statistical trend towards mortality when methicillin-resistant $S$. aureus strains were involved compared with methicillin-sensitive organisms, although the tendency to cause disseminated infection did not prove different according to this bacterial feature (10).

In the meantime, the literature points out a growing role for numerous possible factors supporting invasive staphylococcal disease, such as neonatal age (11), concurrent infectious diseases responsible for a transient immune suppression, like chickenpox (12), and collagen vascular disorders and their treatment (13). Moreover, the toxic shock syndrome (TSS), a multi-organ failure characterized by rapid onset, fever, rash, vomiting, diarrhoea and hypotension, probably linked to the use of tampons during menstruation (14), is probably the most well-known expression of an invasive staphylococcal disease, although TSS-like clinical syndromes have also been described when other pathogens and situations occur. Just the multifactorial pathogenesis of TSS has been linked to the numerous toxic products released during staphylococcal infection (14-18).

On the other hand, a broad spectrum of microbial products, especially bacterial superantigens, have been found to play a potential pathogenetic role in the occurrence of immune-mediated vasculitis and a broad spectrum of autoimmune and immune activation-mediated disorders (16-19). In particular, staphylococcal exotoxins (bacterial superantigens) are well known as potent activators of the immune system, causing a variety of human diseases due to their ability to polyclonally activate T-cells at picomolar concentrations. This activation of both antigen-presenting cells and T-lymphocytes leads to an elevated production of proinflammatory cytokines and T-cell proliferation (18), potentially in an acute, end-organ involvement (i.e. septic shock), but also in the induction of arthritis, glomerulonephritis and other autoimmune diseases (18), as hypothesized in some reliable animal models which were focused only on the study of systemic autoimmunity due to the polyclonally activating effects of staphylococcal products (20). For instance, the $S$. aureus exfoliative toxin responsible for staphylococcal scalded skin syndrome (SSSS) acts in a similar way to autoantibodies against desmoglein 1 which are involved in causing some pemphigous variants (17). A lymphocytosis with an expansion of CD4+/CD8+ double-negative T-lymphocytes was observed in a case of staphylococcal toxic shock syndrome (21): these cells are known to express immune regulatory properties and can increase during certain autoimmune disorders (21).

From a clinical point of view, a broad spectrum of of autoimmune and dysreactive disorders seem to be prompted by the mediation of a bacterial (in particular, staphylococcal) infection. These include systemic lupus erythematosus (22), especially kidney diseases observed during or immediately after $S$. aureus infection (23-25): a case of reversible glomeulonephritis and nephrotic syndrome in a patient with underlying amyloidosis and suffering from methicillin-resistant $S$. aureus acute arthritis (23), five diabetic patients developing an IgAdominant mesangial glomerulonephritis complicated by a poor clinical outcome (24) and one more case of Berger disease (IgA nephropathy) occured after an $S$. aureus septic arthritis and local orthopaedic treatment, with a favourable evolution (25). These last cases of predominantly IgA-mediated nephropathy find their pathogenetic explanation in some accurate ex-vivo studies (26) and animal models (27), which point out 
the crucial role of $S$. aureus cell envelope antigens and their interaction with a predisposed host genetic background in prompting a selective autoimmune activation. Also central nervous system disorders, such as autoimmune encephalomyelitis and multiple sclerosis (28), as well as acute transverse myelopathy (29), may be indirectly associated to $S$. aureus infection, possibly via the proinflammatory activity of bacterial peptidoglycan of these gram-positive cocci, following the stimulation of dendritic cells and the consequent priming of naïve T-lymphocytes (28).

In our case, the community-acquired origin of this multiresistant $S$. aureus infection has to be underlined as a sign of the increasing spread of methicillin-resistant cocci into the environment and the general population $(1,4)$; moreover, our patient did not receive any antimicrobial agents in the previous months. The combination of a diffuse, probably immune-mediate polyvisceritis, with a septicemic pneumonia and probable multiple bacterial embolism involving the liver, spleen, and lungs, is also impressive, as well as the very severe, complicated and prolonged illness. The extensive immune activation status (as demonstrated by a proportionally maintained absolute lymphocyte count and a careful study of T-cell subsets showing an increased quote of $\mathrm{CD} 4+, \mathrm{CD} 34+$, and CD4CD8- cells, compared with a proportional decrease of CD8+ T-lymphocytes) probably had a prominent role in prompting several focal clinical features in our patient, which apparently were not directly related to bacterial invasion, such as the polyvisceritis, the massive pleuric and pericardial effusion, the myocarditis-pericarditis and the thrombophlebitis occurring in multiple and remote body sites, as opposed to the initial pulmonary disease localization. The successful resort to a combination treatment including also the oxazolidinone linezolid (plus rifamicin and tetracycline) represents another key feature, since in our case prior glycopeptide-based associations had an apparently limited clinical effect, despite the maintained in vitro susceptibility to these compounds. Like other reports of severe methicillin-resistant $S$. aureus infections $(5,8)$, the pharmacokinetic and/or pharmacodynamic properties of linezolid (compared with those of glycopeptides) probably played a role in leading to microbiological and subsequently, clinical cure of difficult-to-treat $S$. aureus infections. Finally, the co-administration of corticosteroids probably contributed significantly in containing the immune-mediated disease features, although the time of recovery proved very long. Unfortunately, this issue cannot be discussed extensively, since only anecdotal reports and expert opinion advice are available in the literature $(16,18$ 19, 21, 23-26, 28).

In conclusion, the pathogenetic features of invasive staphylococcal infection need further investigation, including both microbiological aspects (i.e. methicillin- and multi-drug-resistance), and the immunological network and its possible activation triggered by disseminated $S$. aureus disease. The role of novel antimicrobial compounds in the management of methicillin-resistant $S$. aureus strains which remain apparently sensitive to glycopeptides, but do not respond clinically to these last compounds, also deserve controlled studies, as well as steroid administration when an overwhelming immune activation syndrome becomes of concern.

\section{REFERENCES}

1. Decousser J.W., P. Pina, F. Picot, C. Delalande, B. Pangon, P. Courvalin and P. Allouch. 2003. Frequency of isolation and antimicrobial susceptibility of bacterial pathogens isolated from patients with bloodstream infections: a French prospective national survey. J. Antimicrob. Chemother. 51:1213.

2. Stefani S. and P.E. Varaldo. 2003. Epidemiology of methicillin-resistant staphylococci in Europe. Clin. Microbiol. Infect. 9:1179.

3. Manfredi R., A. Nanetti, R. Valentini, L. Calza and F. Chiodo. 2002. A prospective surveillance study of methicillin resistance levels of Staphylococcus aureus strains isolated in selected high-risk wards of a large tertiary care Hospital. Infect. Dis. Clin. Pract. 11:427.

4. Berger-Bachi B. and S. Rohrer. 2002. Factors influencing methicillin resistance in staphylococci. Arch. Microbiol. 178:165.

5. Pistella E., F. Campanile, D. Buongiorno, S. Stefani, G.D. Di Nucci, P. Serra and M. Venditti. 2004. Successful treatment of disseminated cerebritis complicating methicillin-resistant Staphylococcus aureus endocarditis unresponsive to vancomycin 
therapy with linezolid. Scand. J. Infect. Dis. 36:222.

6. Turnidge J. 2003. Impact of antibiotic resistance on the treatment of sepsis. Scand. J. Infect. Dis. 35:677.

7. Moyenuddin M., C.A. Ohl, J.C. Williamson and J.E. Peacock. 2003. Disseminated oxacillin-resistant Staphylococcus aureus infection responsive to vancomycin and quinupristin-dalfopristin combination therapy. J. Antimicrob. Chemother 51:202.

8. Manfredi R., S. Sabbatani and F. Chiodo. 2005. Severe staphylococcal knee arthritis responding favorably to linezolid after glycopeptide-rifampicin failure. A case report and literature review. Scand. $J$. Infect. Dis. 37:513.

9. Manfredi R. 2005. A re-emerging class of antimicrobial agents: streptogramins (quinupristin/ dalfopristin) in the management of multiresistant gram-positive nosocomial cocci in hospital setting. Mini Rev. Med. Chem. 5:1075.

10. Melzer M. S.J. Eykyn, W.R. Gransden and S. Chinn. 2003. Is methicillin-resistant Staphylococcus aureus more virulent than methicillin-susceptible $S$. aureus? A comparative cohort study of British patients with nosocomial infection and bacteremia. Clin. Infect. Dis. $37: 1453$.

11. Healy C.M., D.L. Palazzi, M.S. Edwards, J.R. Campbell and C.J. Baker. 2004. Features of invasive staphylococcal disease in neonates. Pediatrics 114:953.

12. Raja Lope R.J., A.R. Goldstein and J.W. Gray. 2004. Delayed disseminated Staphylococcus aureus infection following chickenpox. J. Paediatr. Child Health 40:320.

13. Liao C.H., T.C. Yao, H.T. Chung, R.I. Lien and J.L. Huang. 2004. Staphylococcal endocarditis and multiple emboli in a patient with systemic lupus erythematosus. J. Rheumatol. 31:2305.

14. Weitz G., H. Djonlagic, P. Montzka, J. Steinhoff and C. Dodt. 2000. Toxic shock syndrome with multi-organ involvement. Dtsch. Med. Wochenschr. 125:1530

15. Floret D. 2001. Clinical aspects of streptococcal and staphylococcal toxinic diseases. Arch. Pediatr. (S)8:762.

16. Hamidou M. and C. Belizna. 2003. Superantigens and vasculitis. Ann. Med. Interne (Paris) 154:96.

17. Amagai M. 2003. Desmoglein as a target in autoimmunity and infection. $J . \mathrm{Am}$. Acad.
Dermatol. 48:244.

18. Krakauer T. 1999. Immune response to staphylocococcal superantigens. Immunol. Res. 20:163.

19. Proft T. and J.D. Fraser. 2003. Bacterial superantigens. Clin. Exp. Immunol. 133:299.

20. Stohl W., D. Xu, S. Zang, K.S. Kim, L. Li, J.A Hanson, S.A. Stohlman, C.S. David and C.O. Jacob. 2001. In vivo staphylococcal superantigendriven polyclonal Ig responses in mice: dependence upon CD4+ cells and human MHC class II. Int. Immunol. 13:1291.

21. Carulli G., G. Lagomarsini, A. Azzara, R. Testi, R. Riccioni and M. Petrini. 2004. Expansion of TcRalphabeta+CD3+CD4-CD8- (CD4/CD8 doublenegative) $\mathrm{T}$ lymphocytes in a case of staphylococcal toxic shock syndrome. Acta Haematol. 111:163.

22. Tomita H., M. Yamada, I. Sekigawa, T. Yoshiike, N. Iida and H. Hashimoto. 2003. Systemic lupus erythematosus-like autoimmune abnormalities induced by bacterial infection. Clin. Exp. Rheumatol. 21:497.

23. Yokota N., H. Morita, S. Iwasaki, H. Ooba, T. Ideura and A. Yoshimura. 2001. Reversible nephrotic syndrome in a patient with amyloid $\mathrm{A}$ amyloidosis of the kidney following methicillinresistant Staphylocococcus aureus infection. Nephron 87:177.

24. Nasr S.H., G.S. Markowitz, J.D. Whelan, J.J. Albanese, R.M. Rosen, D.A. Fein, S.S. Kim and V.D. D'Agati. 2003. IgA-dominant acute poststaphylococcal glomerulonephritis complicating diabetic nephropathy. Hum. Pathol. 34:1235.

25. Pola E., G. Logroscino, V. De Santis, F. Canducei, A. Delcogliano and A. Gasbarrini. 2003. Onset of Berger disease after Staphylococcus aureus infection: septic arthritis after anterior cruciate ligament reconstruction. Arthroscopy 19:29.

26. Koyama A., S. Sharmin, H. Sakurai, Y. Shimizu, K. Hirayama, J. Usui, M. Nagata, K. Yoh, K. Yamagata, K. Muro, M. Kobayashi, K. Ohtani, T. Shimizu and T. Shimizu. 2004. Staphylococcus aureus cell envelope antigen is a new candidate for the induction of IgA nephropathy. Kidney Int. 66:121.

27. Sharmin S., Y. Shimizu, M. Hagiwara, K. Hirayama and A. Koyama. 2004. Staphylococcus aureus antigens induce IgA-type glomerulonephritis 
in Balb/c mice. J. Nephrol. 17:504.

28. Visser L., H. Jan de Heer, L.A. Boven, D. Van Riel, M. Van Meurs, M.J. Melief, U. Zahringer, J. Van Strijp, B.N. Lambrecht, E.E. Nieuwenhuis and J.D. Laman. 2005. Proinflammatory bacterial peptidoglycan as a cofactor for the development of central nervous system autoimmune disease. J. Immunol. 174:808.

29. Ruiz-Ruiz F.J., B. Martin Lorenzo, A. Amores Arriaga, F.J. Ruiz Laiglesia, A.M. Hualde Enguita and J.L. Perez Calvo. 2004. Acute transverse myelopathy associated to $S$. aureus: a difficult differential diagnosis. Neurologia 19:130. 